\title{
Die Frage nach epistemischer Normativität in der Erziehungswissenschaft ${ }^{1}$
}

\author{
Edgar Forster ${ }^{2}$ \\ Universität Fribourg (Schweiz)
}

Wie muss man in politischen Kulturen, in denen die Möglichkeit, Wahrheit zu diskreditieren, nicht ausgeschlossen werden kann, über epistemische Normativität sprechen? Die grundlegende historische Kontingenz aller Wissensansprüche verpflichtet uns, so paradox das erscheinen mag, Objektivität und Realitätsprüfung nicht voreilig als Herrschaftssymbole zu diskreditieren. Was ich unter epistemischer Normativität verhandle, hat ihre Wurzeln in einem Kampf gegen epistemologische, politische und kulturelle Autorität und für einen Wirklichkeitssinn mit all seinen Ambivalenzen und Widersprüchen.

Mein Vortrag hat drei Teile: Erstens werde ich darüber sprechen, wie Sozialwissenschaften Normativität problematisieren. Zweitens argumentiere ich, dass diese Problematisierung mit der Geschichte der europäischen Moderne verknüpft ist, die ihre koloniale Geschichte als „nicht-existent" (Santos 2014) produziert hat. Drittens führen mich Konsequenzen, die ich aus einer dekolonialen Epistemologie ziehe, zur Frage nach der Verortung der Wissensproduktion. Das öffnet den Weg für eine Kritik der Repräsentation.

Unter epistemischer Normativität verstehe ich Regeln, Normen und Wertungen, die die Generierung wissenschaftlichen Wissens steuern und regulieren (Möllers 2015, S. 402f.). Epistemische Normativität bezieht sich nicht nur auf Propositionen, sondern auf den gesamten Prozess der Wissensproduktion, so dass Normen immer auch eine Handlungsdimension aufweisen. Sie erzeugen Handlungsdruck, ohne Handeln zu determinieren (Stemmer 2011). Erziehungswissenschaftliche Wissensproduktion lässt sich als normative Ordnung untersuchen. Bei ihrer Herausbildung und Veränderung spielen externe und interne Faktoren eine wichtige Rolle, und epistemische Normativität umfasst „die internen Perspektiven, Prozesse, Prozeduren und Auseinandersetzungen“ (Forst \& Günther 2011, S. 11). Die Trennung zwischen internen und externen Faktoren ist analytischer Natur; sie ist nützlich, um interne Dynamiken eines wissenschaftlichen Feld in die Analyse einzubeziehen und den Akteursstatus von Wissenschaften erklären zu können. Normative wissenschaftliche Ordnungen bilden den Rahmen für die Rechtfertigung von Ansprüchen auf Wahrheit, Objektivität und wissenschaftliche Redlichkeit. Wie alle Herrschaftsordnungen sind sozialwissenschaftliche Ordnungen konflikthaft und instabil und regeln ihre Machtansprüche durch reflexive Metaprinzipien, Verfahren und Institutionen. Eine letzte Vorüberlegung zu epistemischer Normativität: Nach Möllers (2015, S. 127f.) bestehen Normen aus der Darstellung einer Möglichkeit und einer positiven Bewertung ihrer Verwirklichung. ${ }^{3}$ Man sollte sich also hüten, Normativität 
ausschließlich negativ, als normierende und normalisierende Regeln zu definieren, denen unreglementierte Erfahrungen als Ausdruck unverzerrter Existenzen gegenüber treten.

\section{Normativität in Sozialwissenschaften}

Was problematisieren Sozialwissenschaften, wenn sie epistemische Normativität zum Gegenstand von Kritik und Reflexion machen? Der 1996 publizierte Gulbenkian Report (Wallerstein et al. 1996) befasst sich mit dem globalen Zustand der Sozialwissenschaften, unter anderem mit der Orientierungslosigkeit in grundlegenden Fragen ihres Wissenschaftsverständnisses. Dazu zählen die Ansprüche auf Universalität und Objektivität. Gegenüber beiden Ansprüchen verhält sich der Report ambivalent. Eine Aufgabe der Sozialwissenschaften sei es, systematisches Wissen über die Wirklichkeit zu generieren, das auf eine nachvollziehbare Weise empirisch validiert ist. Forschung dürfe keine Herrschaft über Forschungsergebnisse haben, sie müsse offen für unerwartetes Wissen sein und bestehendes Wissen korrigieren können (ebd., S. 90). Gleichzeitig gebe es aber keine "Neutralität“ in der Wissensproduktion. „Wir stimmen darin überein, dass eine Art fotografischer Repräsentation der sozialen Realität unmöglich ist. Daten sind Wirklichkeitsausschnitte, die immer auf Weltanschauungen und theoretischen Modelle ihrer Zeit beruhen und durch Standpunkte bestimmter Gruppen gefiltert sind. In diesem Sinn unterliegt jeder Ausschnitt einer historischen Konstruktion und verändert sich mit der Veränderung der Welt" (Wallerstein et al. 1996, S. 91). ${ }^{4}$

Da sich der Anspruch auf Objektivitä als vollständige Repräsentation der Welt nicht verteidigen lässt, orientieren sich die Kommissionsmitglieder am Ideal der intersubjektiven Nachvollziehbarkeit (vgl. ebd., S. 92). Damit werden allerdings Fragen wissenschaftlich verbindlicher Regeln und damit verbundener Kämpfe um Hegemonie in Wissenschaftskulturen nicht aufgelöst, sondern nur von Ansprüchen auf vollständige Repräsentation auf methodische Verfahren der Erkenntnisgewinnung verlagert: Im ersten Fall verletzt Standpunktgebundenheit des Wissenssubjekts die vollständige Repräsentation, im zweiten Fall beschränken methodische Normierungen volle Erkenntnis. Immer ist es die der Sozialwissenschaft entgleitende Realität, die sich im Problem der Normativität artikuliert, aber im Gulbenkian Report wird der Status von Realität erstaunlicherweise nicht zum Gegenstand der epistemologischen Reflexion gemacht.

Nicht viel anders wird epistemische Normativität in der deutschsprachigen Erziehungswissenschaft diskutiert. Ein Beispiel von vielen: Breinbauer (2013) untersucht "blinde Flecken“, die das Forschungsfeld "Jugend(kultur)forschung" normativ konstituieren. Als "Leerstellen" bezeichnet sie "die mangelnde Reflexion der impliziten oder implikativen Normativität" (ebd., S. 199), etwa Normierungen durch kategoriale, relationale, prozedurale oder anthropologische Festlegungen (ebd., S. 200). Fehlende Aufmerksamkeit für historische Kontingenz von forschungsrelevanten Themen und leitenden Kategorien begrenzen den Anspruch, "die Realität der Jugend“ (ebd.) zu erfassen, und jede epistemische Normierung verletze das Ideal, die unermesslich reiche Wirklichkeit in ihrer Ganzheit zu bestimmen. Allerdings hebt Breinbauer eine andere Dimension des Forschungsprozesses hervor, schenkt ihr aber in der Folge keine 
Bedeutung: Forscherinnen und Forscher seien an der Herstellung eines Interpretationshorizontes beteiligt, der den „Denkhorizont des Möglichen“ (Ahrens, zit. nach ebd., S. 195) absteckt. Man könnte Normierung in diesem Fall nicht als Verengung des Blicks auf Realität, sondern im Gegenteil als Überschreitung der Faktizität interpretieren oder, im Sinne Möllers, als positive Markierung einer Möglichkeit.

Krinninger und Mülller (2013) argumentieren in die gleiche Richtung. Sie zeigen, „wie der normative Gehalt des Bildungsbegriffes immer dann, wenn man glaubt, ihn einigermaßen kontrollieren oder gar aus der Analyse von Bildungsprozessen heraushalten zu können, an anderer Stelle wieder auftaucht, also gleichsam mit den redlich um normative Offenheit bemühten Forscherinnen und Forschern ,Versteck spielt"“ (S. 58). Auch hier entpuppt sich nicht-normative Forschung als illusionär. Unaufhebbar bleibt die Kluft zwischen Wirklichkeit oder Welt und ihrer Repräsentation durch Wissenschaft. Sie lässt sich nur normativ schließen.

Hans-Rüdiger Müller (2013) nimmt das Thema Normativität im Text „Wertvolle Resultate?" wieder auf, und auf den ersten Blick gibt es eine Kontinuität in der Argumentation. Ausgehend von der Gegenüberstellung zwischen sinnrekonstruierenden und sinnauslegenden Interpretationen soll das Problem bearbeitet werden, "dass wir uns über uns selbst täuschen, wenn wir meinen, uns in normativer Neutralität den Gegenständen des erziehungswissenschaftlichen Interesses zuwenden zu können, während in unserem faktischen Forschungshandeln und unserer Interpretation der Forschungsergebnisse normative Orientierungen unbemerkt ihren Einfluss ausüben" (ebd., S. 40). Ein Stellung nehmendes Moment gegenüber der untersuchten Wirklichkeit könne und solle im Forschungsprozess nicht ausgeschlossen, sondern müsse zum Gegenstand der Reflexion gemacht werden (ebd., S. 41). Ich möchte Müllers Rückgriff auf die Hermeneutik Ricœurs dazu nützen, den von Müller gezogenen Schlussfolgerungen zu erweitern und epistemische Normativität nicht ausschließlich als Repräsentationsproblem zu behandeln.

Soziales Handeln wird bei Ricœur wie textförmiges Material behandelt. In einem ersten Schritt wird die Sinnstruktur und die normative Orientierung, die darin zum Ausdruck kommt und den Akteurinnen und Akteuren der Praxis nicht vollständig bewusst ist, untersucht. Diesen Schritt nennt Ricœur ,Erklärung'. In einem zweiten Schritt - Ricœur nennt ihn ,kritisches Verstehen' - wird die implizite Normativität im Hinblick auf die Bedeutung ausgelegt, die sie außerhalb des Textes hat, das heißt in „jenen Theoriebezügen und Praxisformen, die die pädagogische Aufmerksamkeit auf dieses Dokument begründen“ (Müller 2013, S. 47f.). Das kritische Verstehen schließt die strukturanalytisch entzifferte Normativität mit den normativen Konstruktionen in der Sprache der Pädagogik, in erziehungswissenschaftlichen Theoremen und Argumentationen sowie in öffentlichen Diskursen über Bildung und Erziehung zusammen.

Entscheidend für meine Argumentation ist, dass die Interpretation des Textes eine doppelte epistemologische Operation darstellt. Die Generierung der Sinnstruktur ist das Resultat eines theoretischen Vorgangs, den man als "Realisierung" (Bachelard) und nicht als Entziffern einer Realität bezeichnen müsste. Diese Realisierung wird mit einer zweiten verknüpft, nämlich mit Theoriebezügen und Praxisformen. Die Wissensproduktion findet 
in einem „Zwischen“ statt: Weder geht sie nur aus dem Material hervor, das untersucht wird, noch handelt es sich um eine theoretisch inspirierte Analyse, in der das Material als bloße Illustration dient. Und schließlich ist das ,Material' selbst ein theoretisch produziertes oder aufbereitetes Material. Von der Wissensproduktion, wie sie Müller beschreibt, kann man weder sagen, dass sie beliebig sei, eine bloße Konstruktion, noch dass die theoretische Operation durch das Material oder die Theorien determiniert werde. Weder handelt es sich um rein deskriptive Darstellungsformen noch um normierende oder normalisierende Interpretationen; vielmehr handelt es sich sowohl um deskriptive Aussagen über Strukturen, aber zugleich ist die Darstellung auch performativ in dem Sinne, dass mit der Interpretation etwas realisiert wird, was sich nicht einfach am Material ablesen lässt und auch in anderer Weise realisiert werden könnte. Normative Grenzziehungen können überschritten, modifiziert oder verschoben werden, wie Müller (ebd., S. 48) sagt, aber weder geht es darum, dass Forscherinnen und Forscher sich ihrer Normativität enthalten oder ihre Normen zum Prinzip der Analyse erheben. Es entsteht ein "Zwischenraum", in dem es nicht um Wirklichkeitsrepräsentation geht, sondern um die Frage, was ,realisiert' werden soll. Ich komme darauf zurück.

\section{2. "Von wo aus ich spreche“}

Aus der Sicht einer Epistemology of the South (Santos 2014) sind Humanwissenschaften blind für den Umstand, dass ihr Universalitätsanspruch tief in der Geschichte der europäischen Moderne verwurzelt ist. Selbst elaborierte Kritik sei unfähig zu problematisieren, wie sich Humanwissenschaften zu Subjekten der Kritik machen. Um diese epistemische Beschränkung zu überwinden, brauche es eine Beobachtung dritten Grades, die zeigt, wie sich Beobachterinnen und Beobachter der Wissensordnung als kritische Wissenssubjekte inthronisieren. Erst das würde einen epistemologischen Bruch mit der bestehenden Ordnung bewerkstelligen (Mignolo 2012, S. 123). Die Forderung erscheint unmittelbar einleuchtend, aber die Auffassung, der Beobachtung zweiter Ordnung eine der dritten Ordnung hinzuzufügen, ist noch in der Vorstellung verwurzelt, dass ein immer weiterer Regress die blinden Flecken Forschungsprozessen minimieren könne, so dass das Ziel einer immer totalen Sicht auf die Welt möglich sei. Ein solcher Blick widerspiegelt die in der europäischen Moderne verwurzelte Idee eines „Nullpunktes" ${ }^{5}$ der Geschichte, auf der eine sich selbst transparente Welt gebaut werden könne.

Wenn man diese illusio der Moderne nicht reproduzieren will, genügt es nicht das ist meine Schlussfolgerung - den Ort des Sprechens als Grundlage für eine dekoloniale Geo- und Körperpolitik zu benennen, man muss vielmehr den Begriff der Epistemologie praktisch wenden. Homi Bhabha (1994) nimmt den locus of enunciation in seiner postkolonialen Vernunft nicht als Problematisierung der Repräsentation, sondern als Praxis der Intervention. Er greift dafür auf das Konzept der „minimalen Rationalität“ von Charles Taylor (1985) zurück. Rationalität sei mehr als Vermeidung von Inkonsistenz. Technologische Praktiken verändern die Welt und zwingen uns, unsere Rationalitätsauffassungen in der Auseinandersetzung mit diesen Veränderungen zu entwickeln (ebd., S. 150f.). Für Homi Bhabi (1994) wendet Taylors Rationalitätskonzept 
auf kulturelle Praktiken an. Die Bedeutung von pop culture bestehe darin, dass sie in Kulturen der Moderne intervenieren. Der locus of enunciation erweise sich als eine provokative Praxis; sie repräsentiert nichts, sondern deformiert Bedeutungen, produziert neue und verändert dadurch unser Rationalitätsverständnis. ${ }^{6}$

\section{Zur Kritik der Repräsentation in der Wissensproduktion}

Die Epistemologie der Sozialwissenschaften orientiert sich am Ideal der vollständigen Repräsentation von Wirklichkeit, und Befunde über epistemische Normativität artikulieren regelmäßig Repräsentationskritik. Ein anderer Weg besteht darin, wie Homi Bhabha vorschlägt, Wissensproduktionen jenseits von Repräsentationsmodellen zu denken. Das bringt mich zur historischen Epistemologie von Bachelard, Barad, Hacking, Haraway und anderen. Gemeinsam ist ihnen die Auffassung, dass Forschungspraxis Realität nicht abbildet, sondern komplexe "Realisierungen“ hervorbringt. ${ }^{7}$ Dieser Realismus zweiter Ordnung erzeugt einen „epistemologischen Bruch“. Er bildet einen wichtigen theoretischen Eckpfeiler der historischen Epistemologie. Untersucht werden die historischen und materiellen Bedingungen, unter denen Dinge zu Objekten des Wissens und der Wissenschaft gemacht werden (Rheinberger 2007, S. 11; Foucault 1981).

Haraways Abkehr von einer feministischen Identitätspolitik ${ }^{8}$ geht mit einer Aufmerksamkeit für materiell-kulturelle Praktiken der Wissensproduktion einher. Es sind gleichermaßen Interventionen in die Realität und objektive und verantwortliche Beschreibungen der Welt. ${ }^{9}$ Während der Status des locus of enunciation bei Mignolo undeutlich zwischen geographischer Verortung und theoretischer und politischer Zugehörigkeit angesiedelt ist, versteht Haraway (1995) Verortung als „eine komplexe Konstruktion und keine empirische Aufzählung oder bestimmte Stelle. Verantwortlichkeit hat [...] dann etwas damit zu tun, wie wir unter uns Verbindungen aufbauen, wie wir uns selbst zusammenfügen, und wie wir zusammengefügt sind“ (S. 110).

Verortung, Intervention, Objektivität und Verantwortung sind zentrale Elemente der posthumanistischen Epistemologie und lassen sich mit Karen Barads (2012, 2015) Realismuskonzeption näher bestimmen. Beeinflusst von Niels Bohrs Physikphilosophie ficht sie eine Konzeption von Realität und Dingen an, die der Sprache vorgängig oder, umgekehrt, bloße sprachliche Konstruktionen sind. Stattdessen spricht Barad von Phänomenen, die ein nicht-dualistisches Ganzes darstellen und so unterschiedliche Elemente wie Objekte, Beobachterin oder Beobachter, Messapparate und Theorien umfassen. Sie sind über Intra-aktionen miteinander verbunden, und die Unabhängigkeit der Elemente, zum Beispiel die Unabhängigkeit der Beobachterin von einem gemessenen Objekt, geht aus einem Prozess hervor, den Barad (2012, S. 81) als Abtrennung innerhalb eines Phänomens bezeichnet. Realismus - in der Sprache Barads „agentischer Realismus“ - bezieht sich auf Praktiken innerer Grenzziehungen und Abtrennungen. Dabei handelt es sich um „spezifische Konstruktionen mit realen, materiellen Konsequenzen“ (Barad 2015, S. 49).

Ich möchte Barads Position an einem Beispiel verdeutlichen: Der Begriff ,Kompetenz' lässt sich als Abtrennung innerhalb eines Phänomens - zum Beispiel Bildung oder Lernen - begreifen. Kompetenz entwickelt sich in einem langen, nicht-linearen 
Prozess durch Abtrennung oder Herauslösung aus einem komplexen und keineswegs eindeutig bestimmbaren Phänomen und gewinnt zunehmend eine konsistente materielle Form mit realen und materiellen Konsequenzen. Kompetenz ist weder einfach ein Objekt noch eine Eigenschaft oder Fähigkeit, sondern ein ,Ding', das aus Ideen (über Wissen und Können), Messinstrumenten, Theorien und bildungspolitischen Entscheidungen zusammengefügt ist. Der Prozess der Abtrennung ist die Bedingung der Möglichkeit von Objektivität (Barad 2012, S. 82). Eine Abtrennung ist weder ein willkürlicher noch ein notwendiger Vorgang. Er ist weder nur sozial konstruiert noch unabhängig von einem spezifischen Diskurs (z.B. employability) bestimmbar. Abtrennungen liegen nicht in der alleinigen Macht von Forscherinnen und Forschern, aber sie sind auch nicht auf externe Effekte (z.B. des Politischen) reduzierbar. Die Verantwortung von Wissenschaftlerinnen und Wissenschaftlern besteht darin, für die Konsequenzen ihrer Darstellungsform, das heißt ihrer Abtrennungen einzustehen.

Ich komme noch einmal auf das Beispiel der Interpretation des sozialen Handelns bei Müller (2013) zurück: Nachdem man die Sinnstruktur sozialen Handelns entziffert habe, müsse man die darin eingelagerte Normativität mit „Theoriebezügen und Praxisformen, die die pädagogische Aufmerksamkeit auf dieses Dokument begründen“ konfrontieren, um vom Erklären zu einem kritischen Verstehen zu kommen. Man kann hier nicht umstandslos einen Gegenstand der Forschung unabhängig von theoretischen Operationen von Forscherinnen und Forschern isolieren. Vielmehr schälen sich aus komplexen Relationen, die ein "Zwischen“ schaffen, allmählich Gegenstände des erziehungswissenschaftlichen Wissens heraus und nehmen eine wie immer flüchtige Materialität an. Methodisch kann man diese Strategie als Diffraktion beschreiben (Mazzei 2014). ${ }^{10}$ Im Unterschied zu naturwissenschaftlichen Anordnungen sind komplexe Relationen in den Sozialwissenschaften Interaktionen mit Effekte der Rückkoppelung oder der doppelten Diffraktion, durch die Abtrennungen und die Herausbildung von Objekten oder Dingen immer vom Zerfall bedroht sind (Hacking 1999, S. 56ff.).

\section{Schluss}

Epistemische Normativität gerät immer dann in den Blick, wenn man den Sozialwissenschaften den Vorwurf macht, dass sie in ihren Forschungen Wirklichkeit unvollständig oder verzerrt repräsentieren. Darin verbirgt sich ein Realismus, der nach Piaget (zit. nach Fox Keller 1998, S. 132) im Unterschied zur Objektivität darin besteht, die Existenz des Ich zu ignorieren und die eigene Sichtweise unmittelbar als objektiv und absolut anzusehen. Wenn man sich vom Repräsentationalismus abwendet, verschwindet Normativität als Problem der Epistemologie, denn Wirklichkeitssinn und Objektivität realisieren sich über einen Begriff des Objekts, das sich nicht unabhängig von der Situiertheit und Aufmerksamkeit von Forscherinnen und Forschern, von Messinstrumenten und materiell-semiotischen Diskursen der Wissensproduktion denken lässt. 


\section{Anmerkungen}

1 Manuskript zum gleichnamigen Vortrag, gehalten auf der Tagung „Normativität in der Erziehungswissenschaft" der Sektion „Allgemeine Erziehungswissenschaft“ der Deutschen Gesellschaft für Erziehungswissenschaft (DGfE), 8.-10. März 2017, an der Philipps-Universität Marburg.

${ }^{2}$ Kontakt: Université de Fribourg, Departement Erziehungswissenschaften, Rue P.A. de Faucigny 2, 1700 Fribourg, Schweiz, edgar.forster@unifr.ch.

3 Möllers (2015) definiert Normen nicht nur hinsichtlich der Frage, ob es sich um richtige oder gerechtfertigte Normen handelt, sondern versteht darunter „positiv markierte Möglichkeiten“ (S. 14). Das heißt, Normen verweisen auf einen möglichen Zustand oder auf ein mögliches Ereignis. „Normativität hängt an der Möglichkeit abweichender Weltbeschaffenheit - oder einer Weltbeurteilung, deren Maßstab sich nicht auf die Welt, wie sie ist, beschränkt“ (ebd., S. 14).

4 „We also agree that a quasi-photographic representation of social reality is impossible. All data are selections from reality, based on the worldviews or theoretical models of the era, as filtered through the standpoints of particular groups in each era. In this sense, the bases of selection are historically constructed, and will always inevitably change as the world changes" (Wallerstein et al. 1996, S. 91).

${ }^{5}$ Grosfoguel (2007) definiert diesen Nullpunkt als „the point of view that hides and conceals itself as being beyond a particular point of view, that is, the point of view that represents itself as being without a point of view" (S. 214; vgl. auch Toulmin 1994, S. 281ff.).

6 , $[$ [...] if the epistemological tends towards a reflection of its empirical referent or object, the enunciative attempts repeatedly to reinscribe and relocate the political claim to cultural priority and hierarchy [...] in the social institution of the signifying activity" (Bhabha 1994, S. 254f.). Bhabhas Vorzug von agency gegenüber einer epistemologischen Repräsentationsperspektive artikuliert sich in seinem Konzept des „time lag“. Er beschreibt damit die zeitliche Aufspaltung des kolonialen Diskurses: Spaltung des kolonialen Subjekts, das Gegenstand der Forschung ist, und Spaltung der postkolonialen Theoriebildung (Mignolo 2012, S. 119). Weder auf der Ebene der Forschungspraxis noch auf der Ebene des Gegenstands, des kolonialen Subjekts, gebe es eine Zeitgenossenschaft: "the denial of the denial of coevalness“ (ebd., S. 120).

${ }^{7}$ „Es geht also weniger um die Wirklichkeit und ihre Erkenntnis - um das, was ist - als vielmehr um einen Prozess der ,Verwirklichung', um das, was sein kann" (Rheinberger 2007, S. 39).

${ }^{8}$ Feministische Identitätspolitiken haben die Arbeiten von Adrienne Rich (1984) und anderen beflügelt und in Richs Notes toward a Politics of Location ihren krisenhaften Ausdruck gefunden.

9 „Daher glaube ich, dass mein und ,unser' Problem darin besteht, wie wir zugleich die grundlegende historische Kontingenz aller Wissensansprüche und Wissenssubjekte in Rechnung stellen, eine kritische Praxis zur Wahrnehmung unserer eigenen bedeutungserzeugenden, ,semiotischen Technologien“ entwickeln und einem nicht-sinnlosen Engagement für Darstellungen verpflichtet sein können, die einer ,wirklichen' Welt die Treue halten, einer Welt, die teilweise miteinander geteilt werden kann und unterstützend wirkt auf erdumgreifende Projekte mit einem begrenzten Maß an Freiheit, angemessenem materiellen Überfluss, einer Verminderung der Bedeutung von Leiden und einem begrenzten Maß an Glück“ (Haraway 1995, S. 78f.).

10 „This means that in a diffractive process of data analysis, a reading of data with theoretical concepts (and/or multiple theoretical concepts) produces an emergent and unpredictable series of readings as data and theory make themselves intelligible to one another" (Massei 2014, S. 743). 


\section{Literaturverzeichnis}

Barad, K. (2012). Agentieller Realismus. Frankfurt a. M.: Suhrkamp.

Barad, K. (2015). Verschränkungen. Berlin: Merve.

Bhabha, H. (1994). The location of culture. London: Routledge.

Breinbauer, I. M. (2013). Die (forschungspraxis-)immanente Normativität der Jugend(kultur)forschung. In T. Fuchs, M. Jehle \& S. Krause (Hrsg.), Normativität und Normative (in) der Pädagogik (S. 193-207). Würzburg: Königshausen \& Neumann.

Fox Keller, E. (1998). Liebe, Macht und Erkenntnis. Männliche oder weibliche Wissenschaft? Frankfurt a. M.: Fischer.

Forst, R. \& Günther, K. (2011). Die Herausbildung normativer Ordnungen. Zur Idee eines interdisziplinären Forschungsprogramms. In R. Forst \& K. Günther (Hrsg.), Die Herausbildung normativer Ordnungen. Interdisziplinäre Perspektiven (S. 11-30). Frankfurt a. M.: Campus.

Foucault, M. (1981). Archäologie des Wissens. Frankfurt a. M.: Suhrkamp.

Grosfoguel, R. (2007). The epistemic decolonial turn. Cultural Studies, 21(2-3), 211-223.

Haraway, D. (1995). Die Neuerfindung der Natur. Primaten, Cyborgs und Frauen. Hgg. und eingeleitet von C. Hammer \& I. Stieß. Frankfurt am Main, New York: Campus.

Krinninger, D. \& Müller, H.-R. (2012). Hide and Seek. Zur Sensibilisierung für den normativen Gehalt empirisch gestützter Bildungstheorie. In I. Miethe \& H.-R. Müller (Hrsg.), Qualitative Bildungsforschung und Bildungstheorie (S. 57-75). Opladen: Barbara Budrich.

Mazzei, L. A. (2014). Beyond an Easy Sense: A Diffractive Analysis. Qualitative Inquiry, 20(6), 742-746.

Mignolo, W. D. (2012). Local histories/global designs: Coloniality, subaltern knowledges, and border thinking. Princeton: Princeton University Press.

Möllers, C. (2015). Die Möglichkeit der Normen. Über eine Praxis jenseits von Moralität und Kausalität. Frankfurt a. M.: Suhrkamp.

Müller, H.-R. (2013). „Wertvolle“ Resultate? - Zur Normativität im erziehungswissenschaftlichen Forschungsprozess. In Fuchs, T., Jehle, M. \& Krause, S. (Hrsg.), Normativität und Normative (in) der Pädagogik (S. 39-50). Würzburg: Königshausen \& Neumann.

Rheinberger, H.-J. (2007). Historische Epistemologie zur Einführung. Hamburg: Junius.

Rich, A. (1984). Blood, Bread and Poetry: selected prose 1979-1985. London: Virago Press.

Santos, B. S. (2014). Epistemologies of the South. Justice against epistemicide. Boulder: Paradigm.

Stemmer, P. (2011). Die Konstitution der normativen Wirklichkeit. In R. Forst \& K. Günther (Hrsg.), Die Herausbildung normativer Ordnungen. Interdisziplinäre Perspektiven (S. 57-68). Frankfurt a. M.: Campus.

Taylor, C. (1985). Philosophy and the human sciences. Philosophical papers 2. Cambridge: Cambridge University Press.

Toulmin, S. (1994). Kosmopolis. Die unerkannten Aufgaben der Moderne. Frankfurt a. M.: Suhrkamp.

Wallerstein, I., Juma, C., Fox Keller, E., Kocka, J., Lecourt, D., Mudimbe, V. Y., Mushakoji, K., Prigogine, I., Taylor, P. J., Trouillot, M.-R. (1996). Open the social sciences. Rport of the Gulbenkian Commission on the restructuring of the social sciences. Stanford, CA: Stanford University Press. 